\title{
Endonasal Odontoidectomy in Basilar Invagination
}

\author{
Juan A. Simal-Julián ${ }^{1} \quad$ Pablo Miranda-Lloret ${ }^{1} \quad$ Maria R. Sanchis-Martín ${ }^{2} \quad$ Arnold Quiroz $^{1}$ \\ Carlos Botella-Asunción ${ }^{1}$ Amin B. Kassam ${ }^{3}$ \\ ${ }^{1}$ Neurosurgery Service, Hospital Universitari i Politècnic La Fe \\ Valencia, Valencia, Spain \\ 2 Department of Anaesthesiology, H General Universitario de \\ Valencia, Valencia, Spain
Address for correspondence Juan Antonio Simal-julián, Neurosurgery Service, Hospital Universitari i Politècnic La Fe Valencia, Avda Abril Martorell 106, Valencia 46026, Spain
(e-mail: juanantonio.simal@gmail.com).

${ }^{3}$ Aurora Neuroscience Innovation Institute, Aurora St. Luke's Medical Center, Milwaukee, Wisconsin, United States

J Neurol Surg B 2021;82(suppl S1):S14-S15.

\section{Abstract}

Keywords

- basilar invagination

- endonasal

- endoscope

- odontoidectomy

- transnasal

Objective The endoscopic endonasal odontoidectomy (EEO) is emerging as a feasible surgical alternative to conventional microscopic transoral approach. In this article, we show EEO in the basilar invagination (BI) and describe in detail the technical aspects, advantages, and disadvantages of this approach (-Fig. 1).

Methods We describe EEO using audiovisual material from the neurosurgical department of Hospital Universitari i Politècnic La Fe Valencia database.

Results We present the case of a 61-year-old male patient with Bl. Initially, we performed suboccipital decompression and occipitocervical fusion. Subsequently, after a no significant neurological improvement and persistent anterior compression, EEO was performed. The postoperative evolution was uneventful and the preoperative neurological deficits were recovered rapidly after surgery

Discussion EEO technique enables complete odontoid resection, preventing invasion of aggressive oral bacterial flora, and it is not limited by the mouth opening. As well, it avoids manipulation of the soft palate, therefore evades the risk of velopalatal insufficiency, facilitates immediate oral tolerance, and early extubation. The rostral position of $\mathrm{C} 1-\mathrm{C} 2$ complex in $\mathrm{Bl}$ could suppose a great advantage in favor the endonasal approaches. Mucoperichondrial vascularized flaps could be obtained to avoid a postoperative cerebrospinal fluid (CSF) leak and facilitate the reepithelization process of the surgical bed.

Conclusion EEO may provide a significant anatomic and technical advantage over the trans-oral approach.

The link to the video can be found at: https://youtu.be/Td6MDcjCNKk.
\end{abstract}

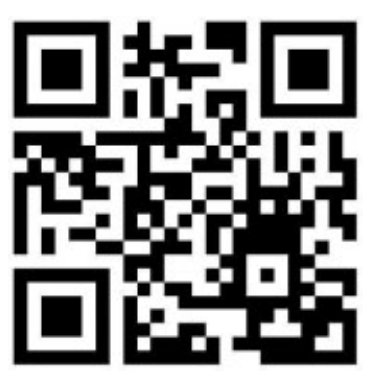

received

February 18, 2019

accepted

March 8, 2020

published online

December 2, 2020

www.thieme.com/skullbasevideos

www.thieme.com/jnlsbvideos

DOI https://doi.org/ $10.1055 / \mathrm{s}-0040-1714406$. ISSN 2193-6331.

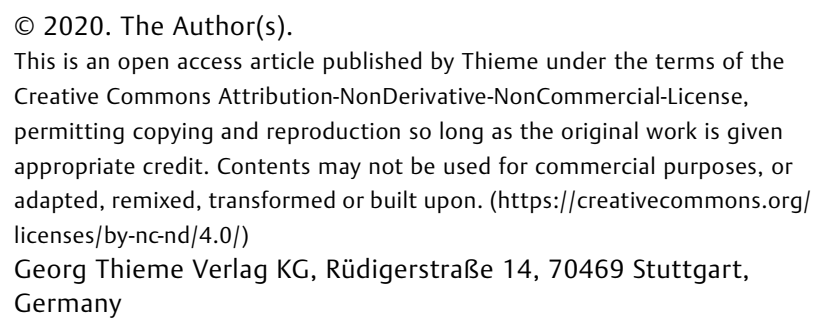




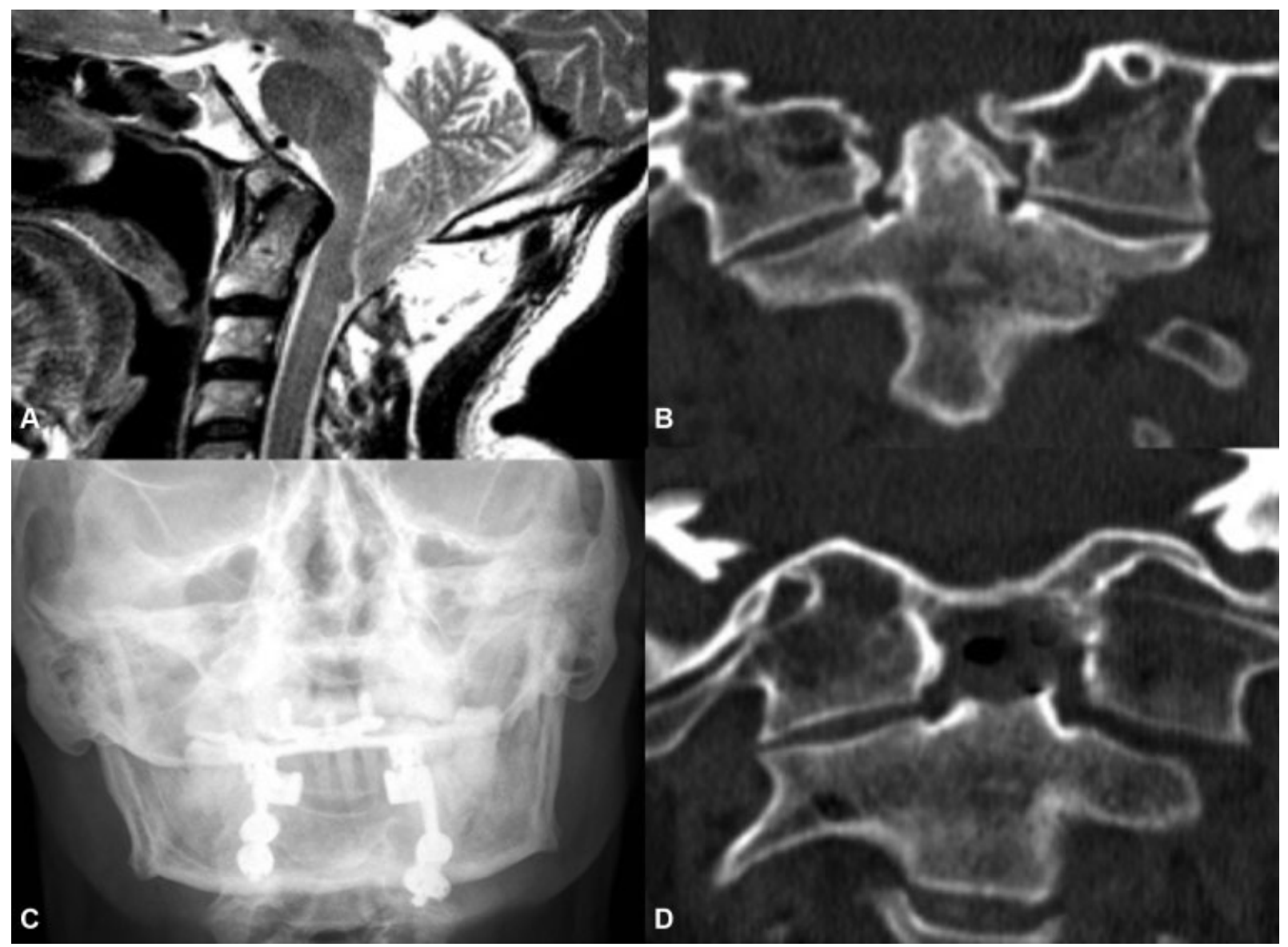

Fig. 1 (A) Preoperative sagittal T2 MRI showing the basilar invagination associated to Chiari malformation. (B) Preoperative coronal CT image showing the assimilation of $\mathrm{C} 1$ to the skull base. (C) X-Ray showing occipitocervical fusion. (D) Postoperative coronal CT image showing odontoid process removed. CT, computed tomography; MRI, magnetic resonance imaging.

Conflict of Interest

A.B.K. is advisory board Medtronic, consultant Synaptive Medical and KLS Martin, and CEO and Founder of Neeka Enterprises.

The rest of the authors certify that they have no affiliations with or involvement in any organization or entity with any financial interest (such as honoraria; educational grants; participation in speakers' bureaus; membership, employment, consultancies, stock ownership, or other equity interest; and expert testimony or patent-licensing arrangements), or nonfinancial interest (such as personal or professional relationships, affiliations, knowledge, or beliefs) in the subject matter or materials discussed in this manuscript. 